\title{
Managing Common Infections in Primary Care: Economic Evaluation of Interventions Targeting Antibiotic Prescribing
}

\section{Fasihul Alam*}

Swansea Centre for Health Economics (SCHE), College of Human and Health sciences, Swansea University, Singleton Park, Swansea SA2 8PP, UK

\section{Introduction}

There have been growing concerns that antimicrobial resistance (AMR) poses major threats to global public health. In Europe alone, it is responsible for about 25,000 hospital deaths caused by selected multidrug resistant infections, leading to an economic burden of 1.5 billion Euros per year [1]. Antibiotic use appears to be an important factor contributing to increase in resistance at the population and individual level [2]. AMR develops rapidly through misuse or over use of antibiotics, which affects patients, families, carers and the society as a whole. Over $80 \%$ of all antibiotic prescribed in the United Kingdom (UK) are in primary care [3], and about a half of these are regarded as unnecessary. Respiratory Tract Infections (RTIs) account for the highest percentage $(60 \%)$ of all antibiotic prescribed in the primary care in the UK [4]. This clearly imposes a huge burden on clinicians and other healthcare services used to manage common infections, e.g. RTIs, Urinary Tract Infections (UTIs), etc.

Targeting antibiotic prescribing at primary care still remains a great challenge for most health care systems in developed countries. Antibiotic prescribing for ambulatory patients decreased substantially during 1990s, however, the use of broad spectrum of antibiotics increased in the United States of America (USA) [5]. In the UK, antibiotic prescribing in primary care has increased since 2000 , for example, there was a $10 \%$ increase in antibiotic prescribing for children between 2003 and 2006 [6].

Antibiotic stewardship is necessary to address unnecessary antibiotic prescribing at the primary care. Only public campaign on antibiotic awareness did not work to reduce antibiotic prescribing in primary care in the UK [7]. Interventions those are multifaceted and broadly applicable to wider population with a range of infections, rather than those involve a single strategy and focus only specific population with specific conditions, appear to be most effective [8]. Behaviour change interventions are likely to be more effective. From a General Practitioner (GP) practice-level large Randomised Control Trial (RCT) in Wales, we indicated that multifaceted educational programme, engaging GPs and patients, can significantly reduce antibiotic consumption at primary care [9]. From a national initiative by the Scottish Antimicrobial Prescribing Group to address antimicrobial stewardship for hospital and primary care prescribing observed significant reduction in antibiotic prescribing with no adverse events on mortality and resistance profile [10]. Recent evidence also suggests to incorporate diagnostic technologies such as point of care test (POCT) to reduce unnecessary antibiotic prescribing in primary care [11]. However, most of the evaluative studies investigating different interventions to reduce antibiotic prescribing suffer from short follow up of patients [12], means that their long-term health and resource implications remain unknown. Although in most trial-based studies the impact of these interventions on patients' resistance profile is overlooked, some studies found a significant reduction in resistance [13].

\section{Current State of Economic Evaluation Evidence}

Economic evidence on the cost-effectiveness of interventions targeting unnecessary antibiotic use is limited and inconclusive. In a recent review study covering both primary and hospital care settings [12], identified only five studies, out of 78 investigated, that incorporated a cost-effectiveness of interventions. However, there are few studies which looked at resource implications of such interventions in primary care setting. Studies that investigated the resource and cost implications mainly focused on a small number of selected items that were affected by interventions. Based on the Stemming the Tide of Antibiotic Resistance (STAR) educational programme for the intervention practices from a RCT [14], we have found that it contributed to $5.5 \%$ reduction in dispensing costs of all-cause oral antibiotics during 12 months follow up, compared to control practices provided usual care. In a primary care study conducted in Norway and Sweden, the cost-effectiveness of a diagnostic test like C-reactive protein POCT showed non-significant reduction in prescribing $(\mathrm{p}=0.08)$ and increased cost $(\mathrm{p}=0.09)$, however it was not clear whether the total costs included all possible resource involved [15].

Although general practitioners (GPs) are aware of the development of antibiotic resistance from their daily clinical practice, however, its impact on healthcare resources and costs are generally overlooked. This is partly due to lack of information on the consequences of antibiotic resistance for the primary care, especially the associated excess healthcare costs [16]. Patients visiting GPs with common infections resistant to one or more common antibiotics prescribed by practitioners are likely to revisit, get additional antibiotics, or may end up with hospitalisations. For example, resistant Escherichia coli UTIs remain symptomatic for longer period and contribute to increase work load in general practice. We recently found that for patients whose E.coli infections were resistant to at least one of the six antibiotics investigated incurred significantly higher antibiotic and reconsultation costs compared to those with sensitive infections [14]. Clearly, this indicates only a conservative estimate of what could be an enormously high. Because, antibiotic resistance can also impose costs on society beyond a health services costs, such as time-off usual work or school. There may also be significant non-monetary costs associated with the longer period of disease-symptoms.

*Corresponding author: M Fasihul Alam, Swansea Centre for Health Economics (SCHE), College of Human and Health sciences, Swansea University, Singleton Park, UK, Tel: +44(0)1792 606502; E-mail: F.alam@swansea.ac.uk

Received December 01, 2015; Accepted December 02, 2015; Published December 06, 2015

Citation: Alam MF (2015) Managing Common Infections in Primary Care: Economic Evaluation of Interventions Targeting Antibiotic Prescribing. Health Econ Outcome Res Open Access 1: e101. doi: 10.4172/2471-268x/1000e101

Copyright: (c) 2015 Alam MF. This is an open-access article distributed under the terms of the Creative Commons Attribution License, which permits unrestricted use, distribution, and reproduction in any medium, provided the original author and source are credited. 
Citation: Alam MF (2015) Managing Common Infections in Primary Care: Economic Evaluation of Interventions Targeting Antibiotic Prescribing. Health Econ Outcome Res Open Access 1: e101. doi: 10.4172/2471-268x/1000e101

The problem with economic evaluation of programmes targeting antibiotic prescribing is that costs of antibiotic resistance are generally ignored in the evaluation. Relevant to this [17], showed from a modelbased study that simply prescribing antibiotics to all patients with UTI symptoms appeared to be more cost-effective than five other alternative strategies with restricted use of antibiotics. The authors, however, mentioned one limitation in that the impact of such a strategy on resistance was excluded from the model. Nevertheless, the estimate of the total cost should include cost of extra treatment of a resistant infection compared with susceptible infection, cost of additional investigations, more expensive drugs, side effects from additional treatments, longer hospital stay, and greater mortality [18].

\section{Conclusion}

The main challenge remains in that, not many trail-based evaluative studies looked at the economic resource implications of the alternative strategies to manage infections in primary care. Those involved an economic evaluation of the alternative strategies suffer from a number of limitations: a short follow up period, not properly investigating health and resource impact of interventions on resistance, not accounting for all relevant resource implications from complications and adverse events due to resistance, etc. Although the cost of a specific infection examined in a study may be relatively small, the overall burden of antibiotic resistance is remarkably high. This is due to the fact that antibiotic resistance can also impose societal (costs of 'out of work', 'out of schools', etc.) and non-monetary costs. Since there is a very limited evidence on how bacteria become resistant with what rate and how specific antibiotic contributes to this process [19], it is really very difficult to accurately measure future health impact and costs incurred from current use of any specific antibiotics targeting common infections in primary care.

\section{Reference}

1. Leung E, Weil DE, Raviglione M (2011) The WHO policy package to combat antimicrobial resistance. Bulletin of the World Health Organization 89: 390-392

2. Costelloe C, Metcalfe C, Lovering A, Alastair D Hay (2010) effect of antibiotic prescribing in primary care on antimicrobial resistance in individual patients: systematic review and meta-analysis.

3. Department of Health (1998) Standing Medical Advisory Committee. The Path of Least Resistance
4. NICE (2008) Respiratory tract infections - antibiotic prescribing, National Institute for Health and Clinical Excellence (NICE) Clinical Guideline 69.

5. Grijalva CG, Nuorti JP, Griffin MR (2009) Antibiotic prescribing rates for acute respiratory infections in US ambulatory settings. JAMA 302: 758-766.

6. Thompson PL, Gilbert RE, Long PF (2009) The effect of antibiotics for otitis media on mastoiditis in children: a retrospective cohort study using the UK General Practice Research Database. Pediatrics 123: 424-430.

7. Huttner B, Goossens H, Verheij T (2010) Characteristics and outcomes of public campaigns aimed at improving the use of antibiotics in outpatients in high-income countries. Lancet Infectious Disease 10:17-31

8. Ranji SR, Steinman MA, Shojania KG (2008) Interventions to reduce unnecessary antibiotic prescribing: a systematic review and quantitative analysis. Med Care 46: 847-862.

9. Butler CC, Simpson SA, Dunstan F (2012) Effetiveness of multifaceted educational programme to reduce antibiotic dispensing in primary care: practice based randomised controlled trial. BMJ 344: 8173

10. Nathwani D, Sneddon J, Pattron A (2012) Antimicrobial stewardship in Scotland impact of a national programme. Antimicrobial Resist Infect Control 3: 7

11. Cooke J, Butler CC, Hopstaken R (2015) Narrative review of primary care point-of-care testing (POCT) and antibacterial use in respiratory tract infection (RTI). BMJ Open Respiratory Research 2: 86.

12. Roque F, Herdeiro MT, Soares S (2014) Educational interventions to improve prescription and dispensing of antibiotics: a systematic review. BMC Public Health 14: 1276

13. Bergman M, Huikko S, Pihlajamäki M, Laippala P, Palva E, et al. (2004) Effect of macrolide consumption on erythromycin resistance in Streptococcus pyogenes in Finland in 1997-2001. Clin Infect Dis 38: 1251-1256.

14. Alam MF, Cohen D, Butler C (2009) Additional costs of antibiotics and reconsultations for antibiotic-resistant Escherichia coli urinary tract infections managed in general practice. International Journal of Antimicrobial Agents 33: 255-257.

15. Oppong R (2013) Cost-effectiveness of point-of-care C-reactive protein testing to inform antibiotic prescribing decision.

16. Coast J, Smith RD, Millar MR (1996) Superbugs: should antimicrobial resistance be included as accost in economic evaluation. Health Economics 5: 217-226

17. Fenwick $\mathrm{EA}$, Briggs $\mathrm{AH}$, Hawke $\mathrm{Cl}$ (2000) Managemnet of urinary tract infection in general practice: a cost-effectiveness analysis. BJGP 50: 635-639

18. Coast J, Smith RD, Karcher AM (2002) Superbugs II: how should economic evaluation be conducted for interventions which aim to reduce antimicrobia resistance. Health Economics 11: 637-647.

19. Turnidge $\mathrm{J}$ (1998) What can be done about resistance to antibiotics: time for action. BMJ 317: 645-647. 